\title{
Volatile compounds from beneficial rhizobacteria Bacillus spp. promote periodic lateral root development in Arabidopsis
}

\author{
Yucong $\mathrm{Li}^{1}$, Jiahui Shao ${ }^{1}$, Yuanming $\mathrm{Xie}^{1}$, Letian $\mathrm{Jia}^{1}$, Yansong $\mathrm{Fu}^{1}$, Zhihui $\mathrm{Xu}^{1}$, Nan \\ Zhang $^{1}$, Haichao Feng ${ }^{1}$, Weibing Xun ${ }^{1}$, Yunpeng Liu ${ }^{2}$, Qi Rong Shen ${ }^{1}$, Wei Xuan ${ }^{1}$, and \\ Ruifu Zhang ${ }^{1}$ \\ ${ }^{1}$ Nanjing Agricultural University \\ ${ }^{2}$ Chinese Academy of Agricultural Sciences
}

November 11, 2020

\begin{abstract}
Lateral root (LR) formation is coordinated by both endogenous and external factors. As biotic factors, Plant growth-promoting rhizobacteria (PGPRs) can affect LR formation, while the regulation mechanism is unclear. In this study, by applying various marker lines, we found that volatile compounds (VCs) from PGPR strain Bacillus amyloliquefaciens SQR9 induced a high frequency of oscillation and prebranch site formation, and further accelerated the development and emergence of the lateral root primordia (LRP), thus promoting LR development in model plant Arabidopsis. We demonstrated a critical role of auxin on SQR9 VCs-induced lateral root formation via respective mutants and pharmacological experiments. Our results showed that the YUC9 (YUCs)-mediated auxin biosynthesis, polar auxin transport, and auxin signaling pathway are involved in SQR9 VCs-induced LRs formation. We further showed that acetoin, a major component of SQR9 VCs, is less active in promoting root development compared to SQR9 cells, suggesting uncharacterized VCs might contribute to SQR9 effect in mediating LR formation. In summary, our study revealed a novel mechanism of PGPR-produced VCs in regulating LR branching in a non-contact manner, and further efforts will explore useful VCs to promote plant root development.
\end{abstract}

\section{INTRODUCTION}

Plant lateral root (LR) development is regulated by both intrinsic and environmental factors. In model plant Arabidopsis, the LR develops along the primary root axis, this process begins when a patch of xylem polepericycle (XPP) cells are specified as LR founder cells (LRFCs), and activated to undergo several anticlinal and periclinal divisions to form a dome-shaped LRP, which finally grows outward and emerges from the primary root (De Rybel et al., 2010; Ive De Smet et al., 2008; Dubrovsky et al., 2008; Fernandez et al., 2015; Malamy \& Benfey, 1997; J. E. Vermeer et al., 2014). Phytohormone auxin plays a critical role in regulating the whole LR development stages (De Rybel et al., 2010; I. De Smet et al., 2007; Du \& Scheres, 2018; Dubrovsky et al., 2008; Lee, Cho, \& Kim, 2015). Recent studies using pDR5:Luciferase reporter line proved that a periodic gene oscillation triggers the repetitive prebranch site formation, and the whole region of rhythmically pulsed expression ofDR5:Luciferase is designated as oscillation zone (OZ) (Moreno-Risueno et al., 2010; Van Norman, Xuan, Beeckman, \& Benfey, 2013). The oscillatory gene expression process is also described as the root clock (W. Xuan, De Gernier, \& Beeckman, 2020), which can be characterized by amplitude and frequency of DR5:Luciferase expression in OZ. Researches further indicated that lateral root cap (LRC)-derived auxin is critical in the output of the root clock through modulating the oscillation amplitude to determine whether a prebranch site is created or not (Moller, Xuan, \& Beeckman, 2017; W. Xuan et al., 2015; W. Xuan et al., 2016).

Environmental factors can affect different LR development stages (Motte, Vanneste, \& Beeckman, 2019). 
Light, heavy metal cadmium, and water deficit has been recently reported to affect LR formation through modulating DR5:Luciferase expression in OZ and subsequent prebranch site formation (Kircher \& Schopfer, 2018; Orman-Ligeza et al., 2018; Xie et al., 2019). Beneficial rhizosphere microorganisms can dramatically affect root development by facilitating root cell division and differentiation (Lopez-Bucio et al., 2007; Ortiz-Castro, Martinez-Trujillo, \& Lopez-Bucio, 2008; Ortiz-Castro, Valencia-Cantero, \& Lopez-Bucio, 2008; Patten \& Glick, 2002; Zamioudis, Mastranesti, Dhonukshe, Blilou, \& Pieterse, 2013; Zou, Li, \& Yu, 2010; Zuniga et al., 2013). Pathogenic bacterium can also strongly induces LR formation (Kong et al., 2020). Moreover, a single bacterial genus (Variovorax ) can manipulate plant hormone levels via metabolic signal interference to maintain root growth in a complex microbiome (Finkel et al., 2020). Nonetheless, how those biotic factors regulate LR formation in spatiotemporal-molding machinery and how they interacted with the intrinsic mechanisms are still not clear.

Bacillus spp., an extracellular plant growth-promoting rhizobacteria (ePGPRs) species, could promote growth of several plant species (Gray \& Smith, 2005; Martinez-Viveros, Jorquera, Crowley, Gajardo, \& Mora, 2010). VCs produced by some Bacillus spp. affect plant growth and development (Meldau et al., 2013; Perez-Flores et al., 2017; Ryu et al., 2003). In this study, we explored the effects of VCs produced by seven different Bacillus spp. strains on plant root development and chose B. amyloliquefaciens SQR9 as the representative to investigate further the role of its VCs in regulating LR development by employing molecular genetics and pharmacological approaches. Our study provides a tentative model for understanding the PGPR manipulating mechanisms on LR formation regulation.

\section{MATERIALS AND METHODS}

\section{Plant material and growth conditions}

Arabidopsis thalianaaccessions Col-0 was as wild-type plant genotypes. The marker linespDR5:GUS (Ulmasov, Murfett, Hagen, \& Guilfoyle, 1997),pDR5:Lucifease(Moreno-Risueno et al., 2010), pCYCB1:GUS (Himanen et al., 2002),PIN1:PIN1:GFP (Benkova et al., 2003), PIN2:PIN2:GFP(Blilou et al., 2005), PIN3:PIN3:GFP (Zadnikova et al., 2010),PIN7:PIN7:GFP (Blilou et al., 2005) and the mutant linestir1afb2

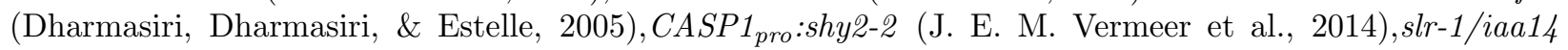
(Fukaki, Tameda, Masuda, \& Tasaka, 2002), arf7arf19 (Yoko Okushima, Fukaki, Onoda, Theologis, \& Tasaka, 2007), aux1-7 (Pickett, Wilson, \& Estelle, 1990), pin2(Roman, Lubarsky, Kieber, Rothenberg, \& Ecker, 1995), pin3(salk_005544), ech2ibr1ibr3ibr10 (Strader et al., 2011), and gh3.3-1gh3.5-2gh3.6-1 (Gutierrez et al., 2012) were used in this study. After 2-3 d of stratification at $4^{\circ} \mathrm{C}$ in the dark,Arabidopsis seeds were surface-sterilized with $30 \%(\mathrm{v} / \mathrm{v}) \mathrm{NaClO}$ solution for $10 \mathrm{~min}$. The seeds were germinated and grown on agar plates containing Murashige and Skoog Basal Salts Mixture (MS salts, PhytoTech LABS) in square Petri plates $(10 \mathrm{x} 10 \mathrm{~cm})$. Standard growth medium consisted of $0.5 \times$ MS salts $\left(2.15 \mathrm{~g} \mathrm{l}^{-1}\right), 0.1 \mathrm{~g} \mathrm{l}^{-1}$ Myo-inositol, $0.5 \mathrm{~g} \mathrm{l}^{-1} 2$-(N-morpholino) ethanesulfonic acid (MES), $1 \%$ sucrose ( $\mathrm{pH} 5.7$ ), and $1 \%$ Agar (Solarbio). Plants were vertically placed in a plant growth chamber, under a long-day photoperiod (16 h: $8 \mathrm{~h}$, light: dark), with a light intensity of $100 \mu \mathrm{mol} \mathrm{m}{ }^{-2} \mathrm{~s}^{-1}$, at $22{ }^{\circ} \mathrm{C}$. After $3 \mathrm{~d}$ of growth, the seedlings were applied for further experiments.

\section{Growth of rhizobacteria and co-cultivation with plants}

For experiments involving bacterial VCs, bi-compartmented Petri dishes (9 $\mathrm{cm}$ diameter) were used. One of the compartments was filled with MSgg (Branda, Gonzalez-Pastor, Ben-Yehuda, Losick, \& Kolter, 2001) agar medium, and the other was filled with MS salts agar medium. Four days before plant experiments, all of the Bacillus spp. strains (obtained from China General Microbiology Culture Collection Center:B. amyloliquefaciens SQR9, B. amyloliquefaciens FZB42,B. amyloliquefaciens SCmB, B. megaterium SXwC, B. megaterium D4, B. subtilis $\mathrm{GZtD}$, B. subilis 168) and SQR9 mutants $\Delta y s n E$ (Shao, Xu, Zhang, Shen, \& Zhang, 2014) and $\Delta$ alsD (Wu et al., 2018) were grown in Luria-Bertani (LB) liquid medium at $37^{\circ} \mathrm{C}$ with $170 \mathrm{rpm}$ overnight. The bacterial cells were washed twice by centrifugation for $3 \mathrm{~min}$ at $5,000 \mathrm{~g}$, and finally resuspended in double-distilled water (DDW). Three drops of $3 \mu \mathrm{l}$ bacterial suspension (or DDW as a control) was spotted at one side of the bi-compartmented Petri dishes filled with MSgg agar. After $3 \mathrm{~d}$ of growth of 
bacterial strains at $28^{\circ} \mathrm{C}$, 3-day-old seedlings (4 plants) were transferred to the adjacent compartment filled with MS salts agar. The plates were sealed with Parafilm and incubated for 6-8 d in a growth chamber at $22^{\circ} \mathrm{C}$. At the end of this period, primary root length, lateral root number, and total biomass were recorded.

To evaluate the effect of $\mathrm{CO}_{2}$ level on the LR formation induced by SQR9 VCs, the uncovered bicompartmented plate containing SQR9 and Arabidopsis was put in a square Petri dish $(12 \times 12 \mathrm{~cm})$ and two flasks with $4 \mathrm{ml} 0.1 \mathrm{M} \mathrm{Ba}(\mathrm{OH})_{2}$ solution were placed in both sides of square Petri dish. A filter paper was inserted into each flask to increase the surface for trapping $\mathrm{CO}_{2}$ (Ditengou et al., 2015) and the square Petri dish was sealed with Parafilm.

\section{Chemical preparation and treatments}

1-N-naphthylphthalamic acid (NPA) and yucasin were dissolved in DMSO to make a $50 \mathrm{mM}$ stock solution. For treatment, the required amount of the stock solutions was added into MS salts agar and mixed in uniform before being poured into Petri dishes. Acetoin ([?] 98\%, Sigma-Aldrich) was dissolved in DDW to make a $250 \mathrm{mM}$ stock solution and was supplied at different doses $(0,10,30,100,300$ and $1000 \mu \mathrm{M})$ to the plant growth medium. Petri dishes containing 5 plants under different treatments were placed in growth chamber for $8 \mathrm{~d}$ to estimate root development. For investigating the effects of acetoin on CYCB1 expression pattern, prebranch sites formation and LRPs development stage, 3-day-old seedlings were grown with $30 \mu \mathrm{M}$ acetoin for $6 \mathrm{~d}$.

To test the effects of other VCs from SQR9 on root development, the pure compounds (dodecane, tetradecane, 2-dodecanone, 2-nonanone, pentadecane, 2-undecanone, 2-heptanone) were purchased from Sigma. Arabidopsisseedlings were exposed to a filter paper added with $0,1,10$ and $100 \mu$ l pure compound in a bi-compartmented Petri dish for $8 \mathrm{~d}$ to estimate root development.

\section{Phenotypic and data analysis}

After co-cultivation with bacteria for an indicated time period, the length of the newly elongated PR grown during the treatment was quantified and emerged LRs in the whole PR were counted. The plates with seedlings were scanned with EPSON XL11000 for the measurement of PR elongation with Fiji software (http://fiji.sc/) and emerged LRs were recorded under a microscope. The LR density was determined by dividing the LR number by the whole PR length for each seedling analyzed. The total biomass production of each seedling was measured on an analytical balance. LRPs were quantified $6 \mathrm{~d}$ after co-cultivation. The pCYCB1:GUS seedlings were stained and cleared to visualize the LRPs at early stages of development and each LRP developmental stages were classified according to Malamy and Benfey (1997) as follows. Stage I, LRP initiation, in the longitudinal plane, approximately 8 to 10 "short" pericycle cells are formed. Stage II, the formed LRP is divided into two layers by a periclinal division. Stage III, the outer layer of the primordium divides periclinally, generating a three-layer primordium. Stage IV, LRP with four cell layers. Stage V-VII, from the LRP is midway through the parent cortex to the LRP appears to be just about to emerge from the parent root.

\section{GUS histochemical staining}

For histochemical analysis of GUS activity, the roots of 3-day-old $p D R 5$ :GUS and pCYCB1:GUS marker lines were incubated overnight at 37 in a GUS reaction buffer after $6 \mathrm{~d}$ of co-cultivation with SQR9. The stained roots were cleared using the method of Malamy and Benfey (1997). For each marker line and

for each treatment, at least 12 transgenic plants were analyzed. A representative sample was chosen and photographed using Leica DM2500 microscope.

\section{Luciferase assay}

pDR5:Lucifease expression along the primary root was analyzed by using Lumazon (Wei Xuan, Opdenacker, Vanneste, \& Beeckman, 2018). After 6-8 d of co-cultivation, pDR5:Lucifease plants were sprayed with a $1 \mathrm{mM}$ potassium luciferin (Gold Biotechnology) and reacted for $15 \mathrm{~min}$ in darkness, then imaged immediately with a 15 min exposure time. For investigating the effects of SQR9 VCs on the periodicity of DR5oscillation, 
the position of root tip was marked after $4 \mathrm{~d}$ of co-cultivation and the prebranch sites formed in the following $2 \mathrm{~d}(48 \mathrm{~h})$ was counted. The picture series were saved as TIFF format by IVScopeEQ software for further analysis in Fiji (http://fiji.sc/). The Fiji lookup tables "Fire" was used to convert black and white images into color scales based on pixel intensity.

\section{Fluorescence microscopy}

For confocal microscopy, control or SQR9 VCs-treated transgenicArabidopsis seedlings ( $p$ PIN1:PIN1:GFP ,pPIN2:PIN2:GFP , pPIN3:PIN3:GFP and pPIN7:PIN7:GFP ) were mounted in DDW on microscope slides. A Leica SP8 laser-scanning microscope was used for fluorescence imaging of the Arabidopsisroots. Chromophores were excited using a 488-nm argon laser, and fluorescence was detected at 500 to $550 \mathrm{~nm}$. More than eight independent seedlings were analyzed per line, and treatment representative images were selected for figure construction.

\section{Analysis of SQR9 VCs}

B. amyloliquefaciens SQR9 were grown in a head-space bottle containing MSgg medium for $6 \mathrm{~d}$ at 28 . The head-space bottle was preheated in a $50{ }^{\circ} \mathrm{C}$ water bath for 5 minutes, then the VCs were collected with Solid-phase microextraction (SPME) fiber (50/30 $\mu \mathrm{m}$ DVB/CAR on PDMS) for 30min. The SPME fiber was removed and desorbed at $180{ }^{\circ} \mathrm{C}$ for 4 minutes. Gas chromatography-mass spectrometry (GC-MS) analysis was carried out by Agilent 7890A-5975V, equipped with a chromatographic column (Agilent DBwax, $30 \mathrm{~m} \times 0.25 \mathrm{~mm} \times 0.25 \mu \mathrm{m})$. Helium was used as the carrier gas $(1 \mathrm{ml} / \mathrm{min})$, and the injection temperature was $260{ }^{\circ} \mathrm{C}$. The column was held at $40{ }^{\circ} \mathrm{C}$ for 5 min and then programmed to increase by $5{ }^{\circ} \mathrm{C}$ per min to a final temperature of $250{ }^{\circ} \mathrm{C}$, which was maintained for $5 \mathrm{~min}$. The interface temperature, ion source temperature and quadrupole temperature was $260{ }^{\circ} \mathrm{C}, 230{ }^{\circ} \mathrm{C}$ and $150{ }^{\circ} \mathrm{C}$ respectively; the ionization mode was EI+, 70ev; the detector voltage was $1106 \mathrm{~V}$; the scanning mode was full-scan, and the mass range is 20-400. These compounds were identified by comparison with mass spectra from a library of NIST 2011 through Agilent MSD ChemStation (E.02.00.493).

\section{RNA extraction, RNA-seq and quantitative real-time PCR analysis}

Plant materials were collected after $4 \mathrm{~d}$ co-cultivation. Frozen samples of the control and SQR9 VCs-treated seedlings were ground in liquid nitrogen and total RNA was extracted with three biological replicates resulting in a total of 6 samples. Experimental protocols for RNA sequence was performed according to the manufacture's (Novogene Experimental Department) technical instruction.

Differential expression analysis of control and SQR9 VCs-treated samples was performed using the DESeq $\mathrm{R}$ package (1.18.0). DESeq provide statistical routines for determining differential expression in digital gene expression data using a model based on the negative binomial distribution. The resulting P-values were adjusted using the Benjamini and Hochberg's approach for controlling the false discovery rate. Genes with an adjusted $\mathrm{P}$-value $<0.05$ found by DESeq were assigned as differentially expressed. The auxin-related differentially expressed genes with a $\mid \log 2$ (FoldChange) $\mid>1$ were selected for further analysis.

Three-day-old Arabidopsis seedlings of Col-0 grown on MS slats agar medium were exposed to SQR9 VCs or not. After $4 \mathrm{~d}$ of co-cultivation, total RNA was isolated from control or SQR9 VCs-treated seedlings using Plant RNA Kit R6827 (OMEGA bio-tek) following the manufacturer's protocol. The first-strand cDNA synthesis was generated from $1 \mu \mathrm{g}$ of total RNA using the HiScript 1st Strand cDNA Synthesis Kit (Vazyme). Quantitative RT-PCR was performed on a StepOnePlus Real-Time PCR System (Applied Biosystems) using an SYBRß Green Master Mix Kit (Vazyme).AtEF1A (AT5G60390) was used as the reference gene and the primers are listed in Supplemental Table S2. The results were obtained from three biological replicates and $2^{-\mathrm{ddCt}}$ was taken for every sample as the relative expression levels. Values detected by quantitative RT-PCR are relative to the lowest value for each gene.

\section{RESULTS}

Bacillusspp. released VCs promoted Arabidopsis LR development 
Arabidopsis seedlings were grown in the presence of 7 differentBacillus strains in bi-compartmented Petri dishes. After $8 \mathrm{~d}$ of co-cultivation, more than half of the bacterial strains, including $B$. amyloliquefaciens SQR9, FZB42, SCmB and B. subtilis168, significantly increased the PR elongation (Fig. 1A and B). A roughly $2^{\sim} 3$ folds increase in LR number was observed in the seedlings treated with all of the strains (Fig. 1C). To exclude the possibility that increased LR number was only caused by longer PR length, we calculated the LR density and the result denied this possibility (Fig 1D). Moreover, except for B. subtilis (GZtD and168), all these strains increased total seedlings biomass production (Fig 1E).

Since B. amyloliquefaciens SQR9 is an excellent PGPR strain (L. Chen et al., 2017; Shao et al., 2014; Wu et al., 2018), we chose SQR9 as a typical model to carry out the following research. We found the regulation of SQR9 VCs on Arabidopsis growth is dose-dependent (Supplemental Fig. S1). All different doses of inoculum can promote PR elongation (Supplemental Fig. S1B). The promotion effect on LR formation and total biomass production per seedling was highest with three drops of $3 \mu \mathrm{l}$ inoculum, while a low bacterial dose (1 drop) or spreading the inoculum on the half of the dishes caused fewer promoting effects (Supplemental Fig. S1, C and D).

To exclude the side-effect of bacterial produced $\mathrm{CO}_{2}$ or auxin for the LR formation induced by SQR9 VCs, we employed $\mathrm{Ba}(\mathrm{OH})_{2}$ to absorb $\mathrm{CO}_{2}$ (Ditengou et al., 2015) and tested an auxin biosynthesis deficient SQR9 mutant strain $\Delta y s n E$ (Shao et al., 2014). These results showed that LR increase is not solely an effect of elevated $\mathrm{CO}_{2}$ levels produced by SQR9 or not a direct effect of auxin from SQR9 (Supplemental Fig. S2). Together, these results demonstrated that the VCs produced byBacillus spp. are practical factors to promote LR formation.

\section{SQR9 released VCs promoted Arabidopsis LR initiation}

To probe the LR development stages that are affected by SQR9 VCs, we used $p C Y C B 1: G U S$ reporter lines to determine the LRP development stages. After $6 \mathrm{~d}$ co-cultivation, the total LRPs number increased by $33 \%$ under SQR9 VCs-treated condition compared to control treatment (Fig. 2B). Intriguingly, LRP stages I-II were notably increased, but LRP stages V-VII were decreased in SQR9 VCs treated seedlings (Fig. 2B). These results indicated that SQR9 VCs increased LR branching might by inducing thede novo formation of LRPs and accelerating the LRP emergence. Besides, we observed an enhanced expression of $C Y C B 1$ in the meristem by SQR9 VCs treatment (Fig. 2D), indicating that SQR9 VCs promote cell division in the root apical meristem. This observation may explain why SQR9 VCs can promote Arabidopsis PR elongation.

Auxin signaling is required for LR initiation (De Rybel et al., 2010; Laskowski et al., 2008; W. Xuan et al., 2020). We detected the auxin response inArabidopsis root tip and LRPs by using pDR5:GUS marker lines. After $6 \mathrm{~d}$ of co-cultivation, we observed an enhanced expression of $p D R 5: G U S$ in the protoxylem at the meristem and also LRPs by SQR9 VCs treatment (Fig. 2, A and C).

\section{SQR9 released VCs affected oscillation and prebranch site formation}

DR5 oscillation in OZ is an inherent mechanism to pre-pattern LR initiation sites along the PR (Laskowski \& Ten Tusscher, 2017; Moreno-Risueno et al., 2010; Van Norman et al., 2013; W. Xuan et al., 2020). Since SQR9 VCs treatment increased LRP number and LR initiation, we wondered whether SQR9 VCs could affect the DR5 oscillation and prebranch site formation. To address this question, we quantified the number of prebranch sites using pDR5:Luciferase reporter lines (Fig. 3A). Compared with control treatment, SQR9 VCs treatment significantly increased the number of prebranch sites by $29 \%$ after $6 \mathrm{~d}$ of co-cultivation (Fig. 3B). Notably, when co-cultivation for a longer time (8 d), SQR9 VCs treatment resulted in a reduction in prebranch site number (Supplemental Fig. S3). The density of the prebranch sites and LR along the whole PR was also increased by SQR9 VCs treatment (Fig. 3D). Moreover, we measured the periodic production of the prebranch sites in $48 \mathrm{~h}$ and found that SQR9 VCs treatment significantly increased the prebranch site number (Fig. 3C), indicating SQR9 VCs-induced prebranch site formed in a higher frequency. Furthermore, we observed enhanced expression of theDR5:Luciferase in the OZ of SQR9 VCs-treated roots (Fig. 3E), indicating an induction of oscillation amplitude by SQR9 VCs. 


\section{Auxin isindispensable for SQR9 VCs-induced LR formation}

LR development is tightly controlled by auxin (Du \& Scheres, 2018). We determined the auxin-related genes expression levels, which had significant changes in RNA-Seq (Supplemental Table S1). After SQR9 VCs treatment, most of the genes had a similar expression trend with that in RNA-seq, and IAA6, IAA19, IAA28 , PIN2 ,PIN3 , YUC9 , GH3.3 were up-regulated and GH3.10 was down-regulated (Fig. 4A). Furthermore, we examined the effects of SQR9 VCs-induced LR formation on a series of auxin related mutants, which are defective in auxin biosynthesis, transport, or signaling. The results showed that in all of the auxin signaling mutants, including tir1afb2, in which oscillation amplitude and prebranch site are drastically compromised (W. Xuan et al., 2015), arf7arf19 and slr-1/iaa14, which completely abolished LR formation (Fukaki et al., 2002; Yoko Okushima et al., 2007), and $C A S P 1_{\text {pro }}$ :shy2-2 , in which a gain-of-function allele of SHOOT HYPOCOTYL2 (SHY2)/IAA3 is restrictedly expressed in the endodermis and LRP emerged is impaired (J. E. Vermeer et al., 2014), SQR9 VCs treatment did not influence LR formation as compared with the control treatment (Fig. 4, B and C). Moreover, even though SQR9 VCs could induce the LR formation in auxin transport mutants (pin2 , pin3, and aux1-7), the effect of promoting LR formation on auxin efflux mutants pin2 and pin3 were weaker than that on Col-0 (Fig. 4, C and D). However, in the auxin influx mutantaux1-7, which has fewer LRs compared with Col-0, SQR9 VCs treatment induced a comparable effect of LR formation to that in Col-0 (Fig. 4, C and D). In mutant gh3.3-1gh3.5-2gh3.6-1, which is defective in auxin conjugation to amino acids (Nakazawa et al., 2001; Staswick et al., 2005), the effects of SQR9 VCs on promoting LR formation had no change compared with Col-0 (Fig. 2, C and D), while in mutant ech2ibr1ibr3ibr10, which is defective in IBA-to-IAA conversion (Strader et al., 2011), SQR9 VCs has an enhanced effects on promoting LR formation compared with that on Col-0 (Fig. 4, C and D). Meanwhile, adding a well-known auxin efflux inhibitor NPA (Kim et al., 2010) to the medium with $2 \mu \mathrm{M}$ repressed the promoting effects of SQR9 VCs on LR formation. When the concentration of NPA reached $5 \mu \mathrm{M}$, the effects of SQR9 VCs on LR formation was disappeared (Fig. 5, A and B). NPA also suppressed SQR9 VCs-promoted prebranch site formation (Fig. 5, $\mathrm{C}$ and D). These observations suggested a functional auxin efflux was required for SQR9 VCs-stimulated LR formation. To confirm this, we analyzed the expression of PIN1, PIN2 ,PIN3 and PIN7 in primary roots of seedlings expressing $p$ PIN1:PIN1:GFP , pPIN2:PIN2:GFP , pPIN3:PIN3:GFP and PIN7:PIN7:GFP . The results showed that SQR9 VCs treatment increased the expression of $p$ PIN2:PIN2:GFP in epidermal and cortex cells, and slightly induced the expression of $P$ PIN3:PIN3:GFP in the stele of primary roots (Fig. 5, $\mathrm{E}$ to $\mathrm{G}$ ), whereas no changes in $p$ PIN1:PIN1:GFP and $p$ PIN7:PIN7:GFPexpression and localization at the root tip was observed when treated with SQR9 VCs (Supplemental Fig. S4).

IAA is mainly synthesized in a two-step pathway from tryptophan (Y. Zhao, 2012). As a rate-limiting step, indole-3-puruvate (IPA) is converted to indole-3-acetic acid (IAA) by the catalysis of flavin monooxygenases encoded by YUCCA (YUC ) genes (Mashiguchi et al., 2011; Y. D. Zhao et al., 2001). To test whether the increase in LR formation was caused by YUCs-mediated auxin biosynthesis under SQR9 VCs treatment, we analyzed the YUCs expression with SQR9 VCs treatment and employed yucasin to inhibit the function of YUCs specifically (Nishimura et al., 2014). In addition to YUC9, the expression of YUC3 , YUC5 , YUC6 , YUC7 were also up-regulated under SQR9 VCs treatment (Fig. 6B). Moreover, the promoting effect of SQR9 VCs on LR formation was repressed with the increasing concentration of yucasin in the medium (Fig. 6, A and C). These results indicated that YUCs -mediated auxin biosynthesis is required for SQR9 VCs-promoted LR formation.

\section{Identification of active chemicals regulating LR formation in SQR9 VCs}

In order to find specific components of SQR9 VCs that promote LR formation, we analyzed the SQR9 VCs ingredients with a solid-phase microextraction (SPME) technique and GC-MS. The result showed that SQR9 produced various classes of compounds; most of them belong to ketones, hydrocarbons, and alcohols (Supplemental Table S3). Since 3-hydroxy-2-Butanone (also known as acetoin) was the most abundant compound within the chromatographic profile of the VCs (Supplemental Table S3), and it has been reported to promote plant growth (Perez-Flores et al., 2017; Ryu et al., 2003). We thus tested the effect of acetoin on LR information inArabidopsis by adding pure acetoin to the medium and using acetoin biosynthesis defective 
mutant $\Delta$ alsD (Wu et al., 2018) in this experiment. The results showed that acetoin slightly promoted LR formation at lower concentration $(10$ and $30 \mu \mathrm{M})$, and higher concentration $(100,300$ and $1000 \mu \mathrm{M})$ has no effects on that (Fig. 7, A and B). The effect of SQR9 VCs on inducing LR formation is mildly repressed when inoculated $\Delta$ alsD (Fig. $7 \mathrm{C}$ and D) and acetoin accounts for $19 \%$ of the effects of SQR9 VCs on promoting LR formation (Fig. 7E). Moreover, the effects of other components detected in SQR9 VCs on LR formation, whilst those pure compounds cannot induce LR formation (Supplemental Table S4). We further investigated whether the mechanism of acetoin promoting LR increase in Arabidopsis is the same as that of SQR9 VCs. Acetoin did not affect the number of prebranch sites formed within 48 hours in DR5:Lucferase seedlings (Fig. 8, C and D) and also not affect the CYCB1 expression pattern at the PR tips (Fig. 8B). Although acetoin slightly increased the total number of LRP, the stage distribution of LRPs was not significantly affected by treatment with it (Fig. 8A). These data indicated that acetoin in SQR9 VCs plays a certain role in promoting LR formation, but it is feeble compared with the effect of SQR9 VCs as a whole and thus not explain the role of SQR9 VCs in promoting LR formation.

\section{DISCUSSION}

In this study, we propose a model in which beneficial rhizobacteriumBacillus spp. released VCs promotes LR branching through modulating oscillation. It increased the frequency of prebranch site formation and further accelerated the emergence of LRP from the PR, resulting in a denser LRP and LR along the PR spatially. This process is dependent on auxin signaling pathway; fully functional machinery of YUCs-mediated auxin biosynthesis and polar auxin transport are also required (Fig. 9).

By detecting the amplitude of the DR5:Luciferase oscillation and counting the numbers of prebranch sites, LRPs and LRs in series of auxin signaling and biosynthesis mutants, W. Xuan et al. (2015) concluded that both aspects of the oscillation, frequency and amplitude, are necessary for LR pre-patterning. In this study, we demonstrated that SQR9 VCs significantly enhanced the signal intensity of the DR5:Luciferaseexpression in the OZ (Fig. 3E) and subsequently formed prebranch sites in shorter period (Fig. 3C). It is suggested that meristem activity can influence the oscillation frequency (Berg \& Tusscher, 2018). Likewise, we observed an enhanced expression of CYCB1 in the meristematic zone of SQR9 VCs-treated seedling (Fig. 2D), indicating that SQR9 VCs promote cell division in the root apical meristem. Moreover, the density of the prebranch sites and LR along the primary root was also increased by SQR9 VCs treatment despite the reduced number of prebranch sites with SQR9 VCs treatment for a longer time (Supplemental Fig. S3 $\mathrm{B}$ and $\mathrm{C}$ ); it is conceivable that denser prebranch sites are the result of higher oscillation frequency while the reduced prebranch site number is due to the accelated LRP outgrowth. Together, we proposed that the enhanced LR pre-patterning should be caused by SQR9 VCs-induced stronger intensity and higher frequency of oscillation.

As a result of oscillation, the spatial distribution of LRP is established, followed by LR initiation, outgrowth, and emergence (W. Xuan et al., 2020). Increased early LRP stages (I-II) and decreased late (V-VII) LRP stages suggested SQR9 VCs induced more LR initiation and probably stimulated LRP emergence in advance (Fig. 1i), and that was further confirmed by the reduced number of prebranch sites with SQR9 VCs treatment for a longer time (Supplemental Fig. S3). The precise molecular mechanism by which SQR9 VCs regulate LRP development is still unclear, whereas histochemical staining of pDR5:GUS in LRPs highlighted the role of local auxin accumulation and signal activation in SQR9 VCs-modulated LRP development (Fig. 2A). The SLR/IAA14-ARF7/ARF19 module, which is an auxin signaling component, is vital for LR initiation. LRP and LRs are utterly absent from slr andarf7arf19 mutant roots (Fukaki, Nakao, Okushima, Theologis, \& Tasaka, 2005; Fukaki et al., 2002; Y. Okushima et al., 2005; Wilmoth et al., 2005). Our results showed SQR9 VCs treatment could not rescue the lack of LRs in slr-1/iaa14 and arf7arf19 mutants (Fig. 2, B to D), and many Aux/IAAs were up-regulated by SQR9 VCs treatment (Fig. 4A; Supplemental Table S1). These data suggest SQR9 VCs-promoted LR initiation requires integral SLR/IAA14-ARF7/ARF19 module, and other Aux/IAAs may have a potential role in this process. Moreover, an endodermal SHOOT HYPOCOTYL2 (SHY2)/IAA3 auxin signaling module, which mediates the endodermal volume loss or shape change, is reported to be crucial for LR initiation (Vermeer \& Geldner, 2015; J. E. Vermeer et al., 2014). Also, a PIN3- 
dependent hormone reflux pathway is necessary for the progress of LRFCs towards the LR initiation phase, and the pin3 mutation caused a decreased LRP development in stage I-II (Q. Chen et al., 2015). In this study, SQR9 VCs did not affect LR formation in CASP1 $1_{\text {pro }}$ :shy2-2 mutant and had a weaker effect on LR formation in pin3 (Fig. 4, B to D), andPIN3 was up-regulated by SQR9 VCs treatment (Fig. 5, E and G; Supplemental Table S1). It is tempting to speculate that SHY2-mediated endodermis deformation and PIN3dependent auxin reflux between the endodermis and pericycle might be involved in SQR9 VCs-promoted LR initiation. Tang et al. (2017) reported that the FUSCA3 (FUS3)-LEAFY COTYLEDON2 (LEC2) complex synergistically activates the expression of the auxin biosynthetic gene $Y U C 4$ in the pericycle founder cells to function in LR initiation. The GH3.3, which encodes acyl-acid-amido synthetases to cope with excessive free IAA inArabidopsis (Gutierrez et al., 2012; Staswick et al., 2005), and YUCs were up-regulated by SQR9 VCs treatment (Fig. 6B; Supplemental Table S1). Moreover, the expression site of up-regulatedDR5:GUS induced by SQR9 VCs, protoxylem pole, is consistent with one of the expression sites of GH3.3 (W. Xuan et al., 2015) (Fig. 2C). Considering the attenuated effect of SQR9 VCs on inducing LR formation with yucasin treatment and the enhanced effect of that on IBA-to-IAA conversion deficient mutant ech2ibr1ibr3ibr10 (Fig. 4, C and D; Fig. 6, A and C), these results indicated the potential roles of YUCs-mediated auxin biosynthesis in SQR9 VCs-induced LR initiation.

Altogether, our study provides a novel spatiotemporal regulation model of B. amyloliquefaciens SQR9produced VCs on the LR formation, which can be instructive to understand how PGPRs or other environment factors reprogramme root architecture. Nevertheless, it remains unclear which chemical in SQR9 VCs play the major role in regulating LR formation. Despite the faint simulative effect of acetoin on LR formation, other substances in SQR9 VCs modulating RSA is worth further exploring.

\section{ACKNOWLEDGMENTS}

This research was funded by the National Natural Science Foundation of China (31822047), the General Financial Grant from the China Postdoctoral Science Foundation (2018M642268), the Fundamental Research Funds for the Central Universities (KJYQ201903, KYT201802 and KJQN201744), Innovative Research Team Development Plan of the Ministry of Education of China (No. IRT_17R56), and Key R\&D Program of Shandong Province (2019JZZY020614).

\section{REFERENCES}

Benkova, E., Michniewicz, M., Sauer, M., Teichmann, T., Seifertova, D., Jurgens, G., \& Friml, J. (2003). Local, efflux-dependent auxin gradients as a common module for plant organ formation. Cell, 115 (5), 591-602. doi:10.1016/s0092-8674(03)00924-3

Berg, T. v. d., \& Tusscher, K. H. t. (2018). Lateral root priming synergystically arises from root growth and auxin transport dynamics.Biorxiv. , 361709. doi:10.1101/361709 \% J bioRxiv

Blilou, I., Xu, J., Wildwater, M., Willemsen, V., Paponov, I., Friml, J., . . . Scheres, B. (2005). The PIN auxin efflux facilitator network controls growth and patterning in Arabidopsis roots. Nature, 433 (7021), 39-44. doi:10.1038/nature03184

Branda, S. S., Gonzalez-Pastor, J. E., Ben-Yehuda, S., Losick, R., \& Kolter, R. (2001). Fruiting body formation by Bacillus subtilis .Proceedings of the National Academy of Sciences of the United States of America, 98 (20), 11621-11626. doi:10.1073/pnas.191384198

Chen, L., Liu, Y., Wu, G., Zhang, N., Shen, Q., \& Zhang, R. (2017). Beneficial rhizobacterium Bacillus amyloliquefaciens SQR9 induces plant salt tolerance through spermidine production. Molecular Plant-Microbe Interactions, 30 (5), 423-432. doi:10.1094/mpmi-02-17-0027-r

Chen, Q., Liu, Y., Maere, S., Lee, E., Van Isterdael, G., Xie, Z., . . . Vanneste, S. (2015). A coherent transcriptional feed-forward motif model for mediating auxin-sensitive PIN3 expression during lateral root development. Nature Communications, 6 (1), 8821. doi:10.1038/ncomms9821 
De Rybel, B., Vassileva, V., Parizot, B., Demeulenaere, M., Grunewald, W., Audenaert, D., . . . Beeckman, T. (2010). A novel aux/IAA28 signaling cascade activates GATA23-dependent specification of lateral root founder cell identity. Current Biology, 20 (19), 1697-1706. doi:10.1016/j.cub.2010.09.007

De Smet, I., Tetsumura, T., De Rybel, B., Frei dit Frey, N., Laplaze, L., Casimiro, I., . . . Beeckman, T. (2007). Auxin-dependent regulation of lateral root positioning in the basal meristem of Arabidopsis .Development, 134 (4), 681-690. doi:10.1242/dev.02753

De Smet, I., Vassileva, V., De Rybel, B., Levesque, M. P., Grunewald, W., Van Damme, D., . . . Beeckman, T. (2008). Receptor-like kinase ACR4 restricts formative cell divisions in the Arabidopsis root.SCIENCE, 322 (5901), 594-597. doi:10.1126/science.1160158

Dharmasiri, N., Dharmasiri, S., \& Estelle, M. (2005). The F-box protein TIR1 is an auxin receptor. Nature, 435 (7041), 441-445. doi:10.1038/nature03543

Ditengou, F. A., Muller, A., Rosenkranz, M., Felten, J., Lasok, H., van Doorn, M. M., . . . Polle, A. (2015). Volatile signalling by sesquiterpenes from ectomycorrhizal fungi reprogrammes root architecture. Nature Communications, 6 , 6279. doi:10.1038/ncomms7279

Du, Y., \& Scheres, B. (2018). Lateral root formation and the multiple roles of auxin. Journal of Experimental Botany, 69 (2), 155-167. doi:10.1093/jxb/erx223

Dubrovsky, J. G., Sauer, M., Napsucialy-Mendivil, S., Ivanchenko, M. G., Friml, J., Shishkova, S., . - . Benkova, E. (2008). Auxin acts as a local morphogenetic trigger to specify lateral root founder cells.Proceedings of the National Academy of Sciences of the United States of America, 105 (25), 8790-8794. doi:10.1073/pnas.0712307105

Fernandez, A., Drozdzecki, A., Hoogewijs, K., Vassileva, V., Madder, A., Beeckman, T., \& Hilson, P. (2015). The GLV6/RGF8/CLEL2 peptide regulates early pericycle divisions during lateral root initiation. Journal of Experimental Botany, 66 (17), 5245-5256. doi:10.1093/jxb/erv329

Finkel, O. M., Salas-Gonzalez, I., Castrillo, G., Conway, J. M., Law, T. F., Teixeira, P. J. P. L., . . . Dangl, J. L. (2020). A single bacterial genus maintains root growth in a complex microbiome. Nature . doi:10.1038/s41586-020-2778-7

Fukaki, H., Nakao, Y., Okushima, Y., Theologis, A., \& Tasaka, M. (2005). Tissue-specific expression of stabilized SOLITARY-ROOT/IAA14 alters lateral root development in Arabidopsis . Plant Journal, 44 (3), 382-395. doi:10.1111/j.1365-313X.2005.02537.x

Fukaki, H., Tameda, S., Masuda, H., \& Tasaka, M. (2002). Lateral root formation is blocked by a gain-offunction mutation in theSOLITARY-ROOT/IAA14 gene of Arabidopsis . Plant Journal, 29 (2), 153-168. doi:10.1046/j.0960-7412.2001.01201.x

Gray, E. J., \& Smith, D. L. (2005). Intracellular and extracellular PGPR: commonalities and distinctions in the plant-bacterium signaling processes. Soil Biology 83 Biochemistry, 37 (3), 395-412. doi:10.1016/j.soilbio.2004.08.030

Gutierrez, L., Mongelard, G., Flokova, K., Pacurar, D. I., Novak, O., Staswick, P., . . . Bellini, C. (2012). Auxin controls Arabidopsis adventitious root initiation by regulating jasmonic acid homeostasis. Plant Cell, 24 (6), 2515-2527. doi:10.1105/tpc.112.099119

Himanen, K., Boucheron, E., Vanneste, S., Engler, J. D., Inze, D., \& Beeckman, T. (2002). Auxinmediated cell cycle activation during early lateral root initiation. Plant Cell, 14 (10), 2339-2351. doi: $10.1105 /$ tpc.004960

Kim, J.-Y., Henrichs, S., Bailly, A., Vincenzetti, V., Sovero, V., Mancuso, S., . . . Nam, H.-G. (2010). Identification of an $\mathrm{ABCB} / \mathrm{P}$-glycoprotein-specific inhibitor of auxin transport by chemical genomics. Journal of Biological Chemistry, 285 (30), 23307-23315. doi:10.1074/jbc.M110.105981 
Kircher, S., \& Schopfer, P. (2018). The plant hormone auxin beats the time for oscillating light-regulated lateral root induction.Development, 145 (23), dev169839. doi:10.1242/dev.169839

Kong, X., Zhang, C., Zheng, H., Sun, M., Zhang, F., Zhang, M., . . . Ding, Z. (2020). Antagonistic interaction between auxin and SA signaling pathways regulates bacterial infection through lateral root in Arabidopsis . Cell Reports, 32 (8), 108060. doi:10.1016/j.celrep.2020.108060

Laskowski, M., Grieneisen, V. A., Hofhuis, H., Hove, C. A., Hogeweg, P., Maree, A. F., \& Scheres, B. (2008). Root system architecture from coupling cell shape to auxin transport. Plos Biology, 6 (12), e307. doi:10.1371/journal.pbio.0060307

Laskowski, M., \& Ten Tusscher, K. H. (2017). Periodic lateral root priming: What makes it tick? Plant Cell, 29 (3), 432-444. doi:10.1105/tpc.16.00638

Lee, H. W., Cho, C., \& Kim, J. (2015). Lateral Organ Boundaries Domain16 and 18 act downstream of the AUXIN1 and Like-Auxin3 auxin influx carriers to control lateral root development inArabidopsis . Plant Physiology, 168 (4), 1792-1806. doi:10.1104/pp.15.00578

Lopez-Bucio, J., Campos-Cuevas, J. C., Hernandez-Calderon, E., Velasquez-Becerra, C., Farias-Rodriguez, R., Macias-Rodriguez, L. I., \& Valencia-Cantero, E. (2007). Bacillus megaterium rhizobacteria promote growth and alter root-system architecture through an auxin- and ethylene-independent signaling mechanism in Arabidopsis thaliana .Molecular Plant-Microbe Interactions, 20 (2), 207-217. doi:10.1094/MPMI-20-20207

Malamy, J. E., \& Benfey, P. N. (1997). Organization and cell differentiation in lateral roots of Arabidopsis thaliana .Development, 124 (1), 33-44.

Martinez-Viveros, O., Jorquera, M. A., Crowley, D. E., Gajardo, G., \& Mora, M. L. (2010). Mechanisms and practical considerations involved in plant growth promotion by rhizobacteria. Journal of Soil Science and Plant Nutrition, 10 (3), 293-319. doi:10.4067/s0718-95162010000100006

Mashiguchi, K., Tanaka, K., Sakai, T., Sugawara, S., Kawaide, H., Natsume, M., . . . Kasahara, H. (2011). The main auxin biosynthesis pathway in Arabidopsis . Proceedings of the National Academy of Sciences of the United States of America, 108 (45), 18512-18517. doi:10.1073/pnas.1108434108

Meldau, D. G., Meldau, S., Hoang, L. H., Underberg, S., Wuensche, H., \& Baldwin, I. T. (2013). Dimethyl disulfide produced by the naturally associated bacterium Bacillus sp B55 promotes Nicotiana attenuata growth by enhancing sulfur nutrition Plant Cell, 25 (7), 2731-2747. doi:10.1105/tpc.113.114744

Moller, B. K., Xuan, W., \& Beeckman, T. (2017). Dynamic control of lateral root positioning. Current Opinion in Plant Biology, 35 , 1-7. doi:10.1016/j.pbi.2016.09.001

Moreno-Risueno, M. A., Van Norman, J. M., Moreno, A., Zhang, J., Ahnert, S. E., \& Benfey, P. N. (2010). Oscillating gene expression determines competence for periodic Arabidopsis root branching.SCIENCE, 329 (5997), 1306-1311. doi:10.1126/science.1191937

Motte, H., Vanneste, S., \& Beeckman, T. (2019). Molecular and environmental regulation of root development. Annual Review of Plant Biology, 70 , 465-488. doi:10.1146/annurev-arplant-050718-100423

Nakazawa, M., Yabe, N., Ichikawa, T., Yamamoto, Y. Y., Yoshizumi, T., Hasunuma, K., \& Matsui, M. (2001). DFL1 , an auxin-responsive GH3 gene homologue, negatively regulates shoot cell elongation and lateral root formation, and positively regulates the light response of hypocotyl length. Plant Journal, 25 (2), 213-221. doi:10.1046/j.1365-313x.2001.00957.x

Nishimura, T., Hayashi, K.-i., Suzuki, H., Gyohda, A., Takaoka, C., Sakaguchi, Y., . . . Koshiba, T. (2014). Yucasin is a potent inhibitor of YUCCA, a key enzyme in auxin biosynthesis. Plant Journal, 77 (3), 352-366. doi:10.1111/tpj.12399 
Okushima, Y., Fukaki, H., Onoda, M., Theologis, A., \& Tasaka, M. (2007). ARF7 and ARF19 regulate lateral root formation via direct activation of $L B D / A S L$ genes in Arabidopsis . Plant Cell, 19 (1), 118-130. doi:10.1105/tpc.106.047761

Okushima, Y., Overvoorde, P. J., Arima, K., Alonso, J. M., Chan, A., Chang, C., . . . Theologis, A. (2005). Functional genomic analysis of the AUXIN RESPONSE FACTOR gene family members inArabidopsis thaliana: Unique and overlapping functions of ARF7 and ARF19 . Plant Cell, 17 (2), 444463. doi:10.1105/tpc. 104.028316

Orman-Ligeza, B., Morris, E. C., Parizot, B., Lavigne, T., Babé, A., Ligeza, A., . . Draye, X. (2018). The xerobranching response represses lateral root formation when roots are not in contact with water.Current Biology, 28 (19), 3165-3173.e3165. doi:https://doi.org/10.1016/j.cub.2018.07.074

Ortiz-Castro, R., Martinez-Trujillo, M., \& Lopez-Bucio, J. (2008). $N$-acyl-L-homoserine lactones: a class of bacterial quorum-sensing signals alter post-embryonic root development in Arabidopsis thaliana . Plant, Cell E Environment, 31 (10), 1497-1509. doi:10.1111/j.1365-3040.2008.01863.x

Ortiz-Castro, R., Valencia-Cantero, E., \& Lopez-Bucio, J. (2008). Plant growth promotion by Bacillus megaterium involves cytokinin signaling. Plant Signaling \& Behavior, 3 (4), 263-265. doi:10.4161/psb.3.4.5204

Patten, C. L., \& Glick, B. R. (2002). Role of Pseudomonas putidaindoleacetic acid in development of the host plant root system.Applied and Environmental Microbiology, 68 (8), 3795-3801. doi:10.1128/aem.68.8.37953801.2002

Perez-Flores, P., Valencia-Cantero, E., Altamirano-Hernandez, J., Pelagio-Flores, R., Lopez-Bucio, J., Garcia-Juarez, P., \& Macias-Rodriguez, L. (2017). Bacillus methylotrophicus M4-96 isolated from maize (Zea mays ) rhizoplane increases growth and auxin content in Arabidopsis thaliana via emission of volatiles.Protoplasma, 254 (6), 2201-2213. doi:10.1007/s00709-017-1109-9

Pickett, F. B., Wilson, A. K., \& Estelle, M. (1990). The aux1mutation of Arabidopsis confers both auxin and ethylene resistance. Plant Physiology, 94 (3), 1462-1466. doi:10.1104/pp.94.3.1462

Roman, G., Lubarsky, B., Kieber, J. J., Rothenberg, M., \& Ecker, J. R. (1995). Genetic analysis of ethylene signal transduction inArabidopsis thaliana : five novel mutant loci integrated into a stress response pathway. Genetics, 139 (3), 1393-1409.

Ryu, C. M., Farag, M. A., Hu, C. H., Reddy, M. S., Wei, H. X., Pare, P. W., \& Kloepper, J. W. (2003). Bacterial volatiles promote growth inArabidopsis . Proceedings of the National Academy of Sciences of the United States of America, 100 (8), 4927-4932. doi:10.1073/pnas.0730845100

Shao, J., Xu, Z., Zhang, N., Shen, Q., \& Zhang, R. (2014). Contribution of indole-3-acetic acid in the plant growth promotion by the rhizospheric strain Bacillus amyloliquefaciens SQR9.Biology and Fertility of Soils, 51 (3), 321-330. doi:10.1007/s00374-014-0978-8

Staswick, P. E., Serban, B., Rowe, M., Tiryaki, I., Maldonado, M. T., Maldonado, M. C., \& Suza, W. (2005). Characterization of anArabidopsis enzyme family that conjugates amino acids to indole-3-acetic acid. Plant Cell, 17 (2), 616-627. doi:10.1105/tpc.104.026690

Strader, L. C., Wheeler, D. L., Christensen, S. E., Berens, J. C., Cohen, J. D., Rampey, R. A., \& Bartel, B. (2011). Multiple facets of Arabidopsis seedling development require indole-3-butyric acid-derived auxin. Plant Cell, 23 (3), 984-999. doi:10.1105/tpc.111.083071

Tang, L. P., Zhou, C., Wang, S. S., Yuan, J., Zhang, X. S., \& Su, Y. H. (2017). FUSCA3 interacting with LEAFY COTYLEDON2 controls lateral root formation through regulating YUCCA4 gene expression inArabidopsis thaliana . New Phytologist, 213 (4), 1740-1754. doi:10.1111/nph.14313

Ulmasov, T., Murfett, J., Hagen, G., \& Guilfoyle, T. J. (1997). Aux/IAA proteins repress expression of reporter genes containing natural and highly active synthetic auxin response elements. Plant Cell, 9 (11), 
1963-1971. doi:10.1105/tpc.9.11.1963

Van Norman, J. M., Xuan, W., Beeckman, T., \& Benfey, P. N. (2013). To branch or not to branch: the role of pre-patterning in lateral root formation. Development, 140 (21), 4301-4310. doi:10.1242/dev.090548

Vermeer, J. E., \& Geldner, N. (2015). Lateral root initiation inArabidopsis thaliana : a force awakens. F1000Prime Reports, 7 , 32. doi:10.12703/P7-32

Vermeer, J. E., von Wangenheim, D., Barberon, M., Lee, Y., Stelzer, E. H., Maizel, A., \& Geldner, N. (2014). A spatial accommodation by neighboring cells is required for organ initiation inArabidopsis . SCIENCE, 343 (6167), 178-183. doi:10.1126/science.1245871

Vermeer, J. E. M., von Wangenheim, D., Barberon, M., Lee, Y., Stelzer, E. H. K., Maizel, A., \& Geldner, N. (2014). A spatial accommodation by neighboring cells is required for organ initiation inArabidopsis . SCIENCE, 343 (6167), 178-183. doi:10.1126/science.1245871

Wilmoth, J. C., Wang, S. C., Tiwari, S. B., Joshi, A. D., Hagen, G., Guilfoyle, T. J., . . Reed, J. W. (2005). NPH4/ARF7 and ARF19 promote leaf expansion and auxin-induced lateral root formation. Plant Journal, 43 (1), 118-130. doi:10.1111/j.1365-313X.2005.02432.x

Wu, G., Liu, Y., Xu, Y., Zhang, G., Shen, Q., \& Zhang, R. (2018). Exploring elicitors of the beneficial rhizobacterium Bacillus amyloliquefaciens SQR9 to induce plant systemic resistance and their interactions with plant signaling pathways. Molecular Plant-Microbe Interactions, 31 (5), 560-567. doi:10.1094/mpmi11-17-0273-r

Xie, Y., Wang, J., Zheng, L., Wang, Y., Luo, L., Ma, M., . . Xuan, W. (2019). Cadmium stress suppresses lateral root formation by interfering with the root clock. Plant, Cell $\mathscr{E}$ Environment, 42 (12), 3182-3196. doi:10.1111/pce.13635

Xuan, W., Audenaert, D., Parizot, B., Moller, B. K., Njo, M. F., De Rybel, B., . . Beeckman, T. (2015). Root cap-derived auxin pre-patterns the longitudinal axis of the Arabidopsis root.Current Biology, 25 (10), 1381-1388. doi:10.1016/j.cub.2015.03.046

Xuan, W., Band, L. R., Kumpf, R. P., Van Damme, D., Parizot, B., De Rop, G., . . B Beeckman, T. (2016). Cyclic programmed cell death stimulates hormone signaling and root development in Arabidopsis .SCIENCE, 351 (6271), 384-3877. doi:10.1126/science.aad2776

Xuan, W., De Gernier, H., \& Beeckman, T. (2020). The dynamic nature and regulation of the root clock. Development, 147 (3), dev181446. doi:10.1242/dev.181446

Xuan, W., Opdenacker, D., Vanneste, S., \& Beeckman, T. (2018). Long-term in vivo imaging of luciferasebased reporter gene expression in Arabidopsis roots. Methods in molecular biology (Clifton, N.J.), 1761 , 177-190. doi:10.1007/978-1-4939-7747-5_13

Zadnikova, P., Petrasek, J., Marhavy, P., Raz, V., Vandenbussche, F., Ding, Z., . . . Benkova, E. (2010). Role of PIN-mediated auxin efflux in apical hook development of Arabidopsis thaliana .Development, 137 (4), 607-617. doi:10.1242/dev.041277

Zamioudis, C., Mastranesti, P., Dhonukshe, P., Blilou, I., \& Pieterse, C. M. (2013). Unraveling root developmental programs initiated by beneficial Pseudomonas spp. bacteria. Plant Physiology, 162 (1), 304-318. doi:10.1104/pp.112.212597

Zhao, Y. (2012). Auxin biosynthesis: a simple two-step pathway converts tryptophan to indole-3-acetic acid in plants. Molecular plant, 5 (2), 334-338. doi:10.1093/mp/ssr104

Zhao, Y. D., Christensen, S. K., Fankhauser, C., Cashman, J. R., Cohen, J. D., Weigel, D., \& Chory, J. (2001). A role for flavin monooxygenase-like enzymes in auxin biosynthesis. SCIENCE, 291 (5502), 306-309. doi:10.1126/science.291.5502.306 
Zou, C., Li, Z., \& Yu, D. (2010). Bacillus megaterium strain XTBG34 promotes plant growth by producing 2-pentylfuran. The Journal of Microbiology, 48 (4), 460-466. doi:10.1007/s12275-010-0068-z

Zuniga, A., Poupin, M. J., Donoso, R., Ledger, T., Guiliani, N., Gutierrez, R. A., \& Gonzalez, B. (2013). Quorum sensing and indole-3-acetic acid degradation play a role in colonization and plant growth promotion of Arabidopsis thaliana by Burkholderia phytofirmans PsJN. Molecular Plant-Microbe Interactions, 26 (5), 546-553. doi:10.1094/MPMI-10-12-0241-R

\section{Hosted file}

Figures.pdf available at https://authorea.com/users/374969/articles/492344-volatilecompounds-from-beneficial-rhizobacteria-bacillus-spp-promote-periodic-lateral-rootdevelopment-in-arabidopsis 